\title{
miR-24 affects hair follicle morphogenesis targeting Tcf-3
}

\author{
I Amelio ${ }^{1,2}$, AM Lena $^{2}$, E Bonanno ${ }^{3}$, G Melino $^{*, 1,2}$ and E Candi*,2
}

During embryonic development, hair follicles (HFs) develop from an epidermal-mesenchymal cross talk between the ectoderm progenitor layer and the underlying dermis. Epidermal stem cell activation represents a crucial point both for HF morphogenesis and for hair regeneration. miR-24 is an anti-proliferative microRNA (miRNA), which is induced during differentiation of several cellular systems including the epidermis. Here, we show that miR-24 is expressed in the HF and has a role in hair morphogenesis. We generated transgenic mice ectopically expressing miR-24 under the K5 promoter. The K5::miR-24 animals display a marked defect in HF morphogenesis, with thinning of hair coat and altered HF structure. Expression of miR-24 alters the normal process of hair keratinocyte differentiation, leading to altered expression of differentiation markers. MiR-24 directly represses the hair keratinocyte stemness regulator Tcf-3. These results support the notion that microRNAs, and among them miR-24, have an important role in postnatal epidermal homeostasis.

Cell Death and Disease (2013) 4, e922; doi:10.1038/cddis.2013.426; published online 14 November 2013

Subject Category: Experimental Medicine

The epidermis derives from a single layer of ectoderm progenitor, the periderm, which during development gives rise to a pluristratified and keratinized tissue (for review, see Candi et al. ${ }^{1}$ ). During embryogenesis, starting at E14-E15 of mouse embryonic development, the ectoderm layer forms several invaginations, termed placodes, which, growing progressively, form the hair follicles (HF). The morphogenesis of the HFs requires several epithelial-mesenchymal interactions. In the early stage, mesenchymal cells condensate to form the dermal papilla (DP). DP has the ability to promote proliferation and drive differentiation required for $\mathrm{HF}$ development (for review, see Blanpain and Fuchs ${ }^{2}$ ). Briefly, the hair gem extends downward and enwraps the DP that give rise to the hair matrix. When differentiation starts proliferating, hair matrix cells generate the inner root sheath (IRS), which represents the envelope for the future hair shaft and specifically expresses different proteins, such as trichohyalin (AE15). ${ }^{3-5}$ The outer layer of cells, which are in direct contact with the dermis, become the outer root sheath (ORS). The ORS is continuous with the basal layer of the epidermis and expresses $\mathrm{K} 5$ and $\mathrm{K} 14$, similar to the basal compartment of the interfollicular epidermis. After birth, HFs of postnatal (P) day 16 mice reach their full maturation and start an apoptosisdependent phase of regression, known as catagen., ${ }^{6,7}$ The major part of the HF undergoes rapid degeneration, whereas only the bulge region remains just in contact with the DP. The bulge represents the permanent component of $\mathrm{HF}$ that constitutes the HF stem cell reservoir. After a few days of quiescence (telogen), an epidermal-mesenchymal cross talk induces the activation of some stem cells followed by a phase of growth (anagen) that leads the HF in a new state of full maturation.

Wnt/ $\beta$-catenin signaling is crucial for the first stage of HF morphogenesis, and, indeed, conditional ablation of $\beta$-catenin or constitutive expression of a soluble Wnt inhibitor (Dkk1) results in the absence of placode formation. ${ }^{8,9}$ In response to Wnt, $\beta$-catenin can associate with Tcf $\backslash$ Lef 1 transcription factor family and modulate the expression of downstream target genes. Lef1 represents another main effector of this pathway in HF morphogenesis; indeed, Lef1-null mice lack hair coat, and transgenic mice overexpressing inactive forms of Lef1 present severe alteration of differentiation and HF cell fate. ${ }^{10-14}$ Recently, it has been shown that aberrant signaling by TCF/Lef1 during tissue regeneration can result in the development of ectopic sebaceous glands originating from bulge cells. ${ }^{15}$ Tcf-3 is a transcriptional repressor expressed in progenitors of the developing epidermis, whereas in adult epidermis it is confined to stem cells of HF 'bulge' region or embryonic developing ectoderm. ${ }^{16,17}$ Tcf-3 gene ablation results in embryonic lethality with partial axis duplication and ectopic mesoderm. ${ }^{18}$ In adult epidermis, Tcf-3 acts as a crucial factor for the maintenance of skin progenitor cells in the undifferentiated state. Overexpression of Tcf-3 in the epidermis results in the accumulation of $\mathrm{K} 5$ and Sox 9 in keratinocytes with undifferentiated morphology, with a global inhibition of differentiation. The mechanism of Tcf-3 gene regulation during development is still unclear.

Several microRNAs (miRNAs) are expressed in the epidermis, ${ }^{19-24}$ although their role is still partly unexplored. Interestingly, in newborn mice carrying an epidermal-specific

\footnotetext{
${ }^{1}$ Medical Research Council, Toxicology Unit, Leicester University, Leicester LE1 9HN, Leicester, UK; ${ }^{2}$ Biochemistry Laboratory IDI-IRCCS and University of Rome 'Tor Vergata', Department of Experimental Medicine and Biochemical Sciences, Rome 00133, Italy and ${ }^{3}$ Department of Biopathology and Image Diagnostics, University of 'Tor Vergata', Rome 00133 , Italy

${ }^{*}$ Corresponding authors: G Melino or E Candi, Department of Experimental Medicine and Biochemical Sciences, University of Rome, via Montpellier 1, Rome 00133, Italy. E-mails: melino@uniroma2.it or candi@uniroma2.it

Keywords: microRNA; miR-24; hair follicle; skin; Tcf-3; stem cells

Abbreviations: miR or miRNA, microRNA; DP, dermal papilla; HF, hair follicle; K, keratin; HEK, human epidermal keratinocytes; BrdU, 5-bromodeoxyuridine; ORS, outer root sheath; IRS, inner root sheath; $E$, stage of embryonic development

Received 19.7.13; revised 25.9.13; accepted 30.9.13; Edited by RA Knight
} 
Dicer deletion, HFs were stunted and hypoproliferative, hairshaft and inner-root-sheath differentiation was initiated, but the mutant HFs were misoriented, ${ }^{20}$ suggesting that processing of miRNAs and their assembly into the RNA-induced silencing complex is necessary for hair-follicle biology. In addition, miRNAs are important also in maintaining the ability of adult HFs in normal cycles of growth and regression. ${ }^{25,26}$ MicroRNA-24 (miR-24) is an abundant miRNA, which is highly conserved in different species; it is clustered with two other miRNAs, miR-23 and miR-27, on chromosome 9 (cluster-1: miR-23b, miR-27b and miR-24-1) and on chromosome 19 (cluster-2: miR-23a, miR-27a and miR-24-2). We have recently reported that miR-24 drives the differentiation of interfollicular keratinocytes by controlling the actin cable dynamics and cell polarity required during differentiation and stratification of the epithelia. $^{27}$ Alteration of keratinocyte cytoskeleton architecture affects epidermal differentiation, leading to developmental defects or cancer. MiR-24 is upregulated in several cell types, including post-mitotic differentiation of hematopoietic cell lines, ${ }^{28}$ and differentiating neurons $^{29,30}$ and myoblasts. ${ }^{31,32}$ miR-24 suppresses the expression of crucial cell-cycle regulators, E2F2 ${ }^{33,34}$ and Myc, ${ }^{35,36}$ via binding to seedless $3^{\prime}$-UTR recognition elements. $^{28}$

Here, we investigated the involvement of miR-24 in hair development, using transgenic mice, which ectopically express miR-24 under the control of the keratin $\mathrm{K} 5$ promoter. The mice develop severe impairment of hair growth, associated with a reduced proliferation in hair matrix and an altered differentiation. MiR-24 showed, both in vitro and in vivo, the ability to directly target the undifferentiated state regulator Tcf-3. Our data suggest a possible novel role for miR-24 in promoting the HF development by repressing Tcf-3.

\section{Results}

Mir-24 is expressed in the differentiated compartment of HFs. MiR-24 induction is associated with differentiation in several cell types and organs. ${ }^{28,29,31}$ MiR-24 has been reported among the ten most abundant miRNAs expressed in newborn mouse whole skin, ${ }^{20}$ and more specifically it is particularly abundant in mouse HFs. ${ }^{37}$ We have previously reported that miR-24 is mainly confined in differentiated compartments of the interfollicular epidermis. ${ }^{27}$ To study the role of miR-24 in HF morphogenesis, we first analyzed its expression and localization in this specific epidermal compartment. We found that miR-24 is highly expressed in the mouse HFs. Undifferentiated cells of the ORS and matrix showed lower miR-24 signal, whereas a strong signal was evident in IRS (Figure 1). The analysis therefore suggested that miR-24 is expressed in the HF, and it is mostly confined to the differentiated compartment of P8 mouse HFs. Further miR-24 expression analysis in HFs at different developmental and adult stages was not possible owing to the severe phenotype of the transgenic mice. ${ }^{27}$

MiR-24 ectopic expression in mouse epidermis causes defective HF morphogenesis. We then analyzed the phenotype of $\mathrm{Tg} ; \mathrm{K} 5:: \mathrm{miR}-24$ transgenic mice (Figure 2). K5 promoter constitutively leads to the expression of miR-24 in

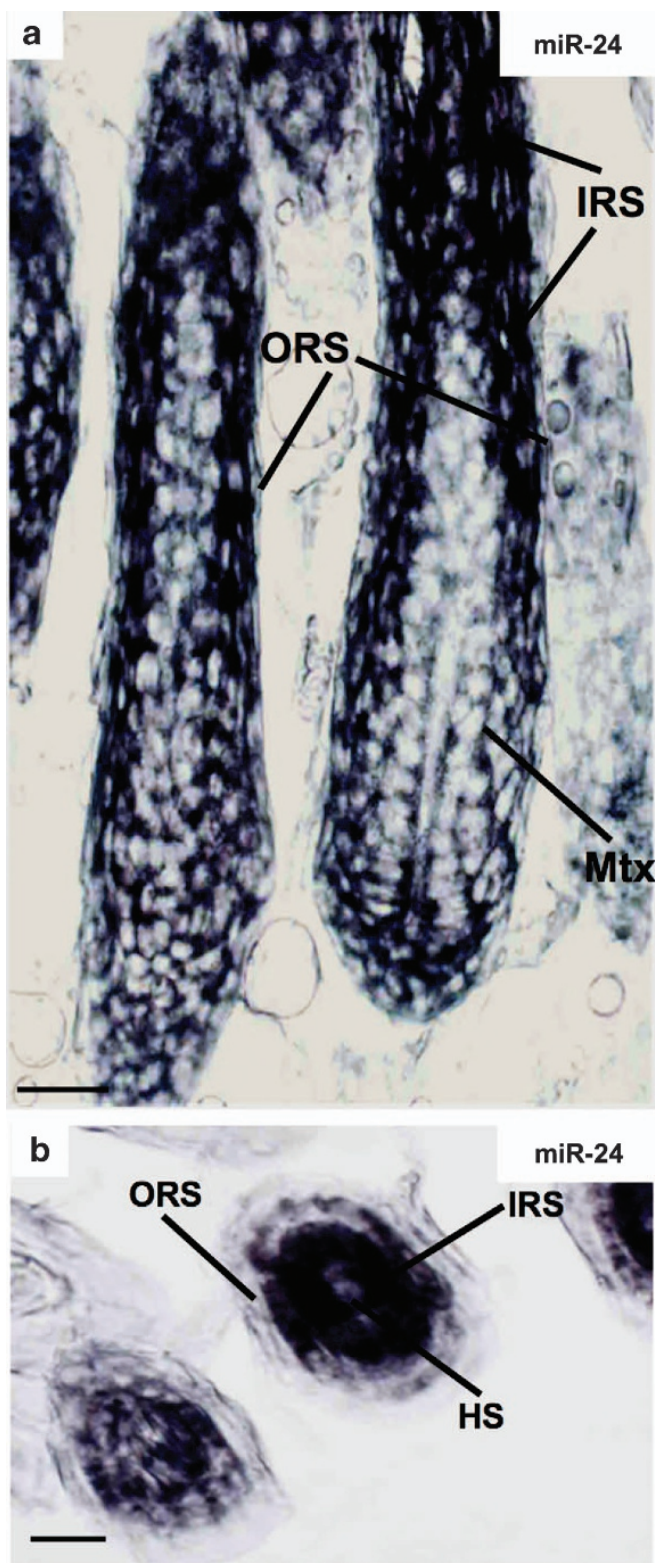

Figure 1 miR-24 is confined in differentiated layers of the hair follicle. (a and $\mathbf{b}$ ) In situ hybridization on P8 mouse back skin, showing high expression of miR-24 in IRS. IRS, inner root sheet; HS, hair shaft; ORS, outer root sheet. Bar in a indicates $100 \mu \mathrm{m}$; bar in $\mathbf{b}$ indicates $50 \mu \mathrm{m}$

the basal compartment of the interfollicular epidermis and in ORS of HFs. As previously reported, $90 \%$ of the Tg;K5::miR-24 newborn mice died shortly after birth, according to severe phenotypes of epidermal barrier impairment. ${ }^{27}$ The Tg;K5::miR-24 mice presented evident thinning of the hair coat (Figure 2b), compared with the wt age-matched littermates. Some sporadic transgenic mice with low phenotype severity appeared to develop normal hair coat, showing several spots in which hair was absent (Figure 2c). In P3 $\mathrm{Tg} ; \mathrm{K} 5:: \mathrm{miR}-24$ mice, the density of HFs was significantly reduced, as assessed by counting the number of HFs per $\mathrm{mm}^{2}$ of the epidermis ( $40 \%$ reduction, Figures $2 \mathrm{~d}$ and $\mathrm{e}$ ). Histological analysis of P3 mouse back skin showed that Tg;K5::miR-24 HFs appeared strongly hypotrophic: 


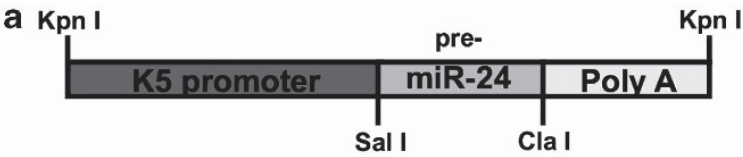

b

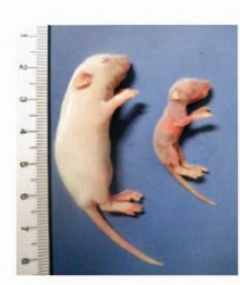

e
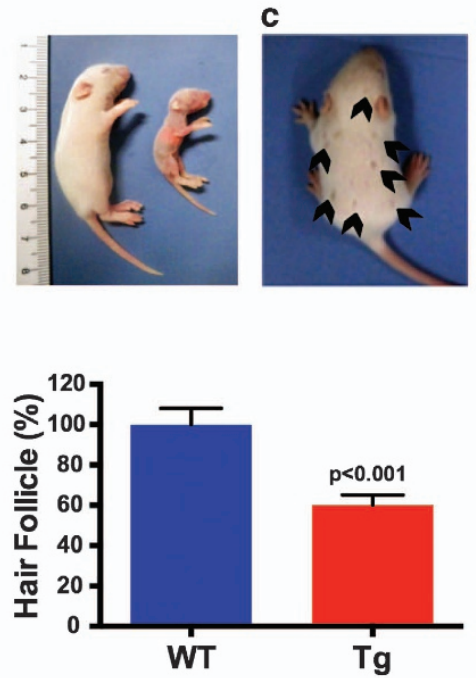

d
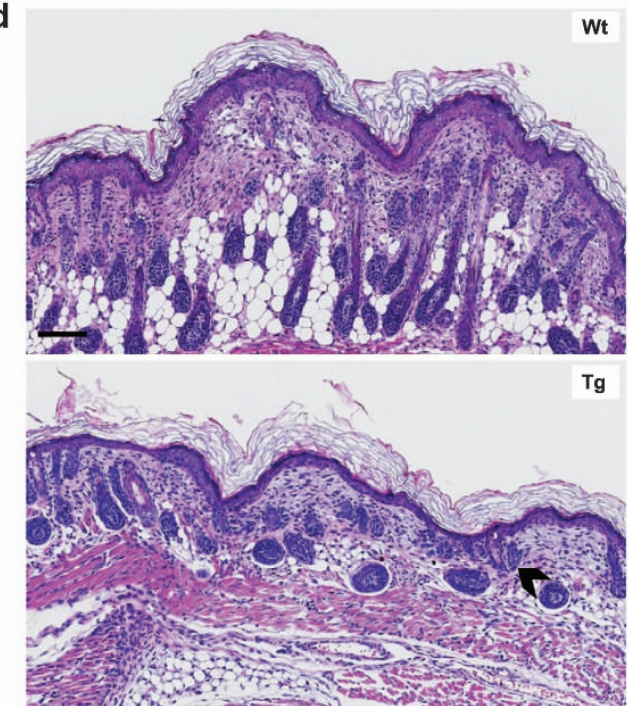

f

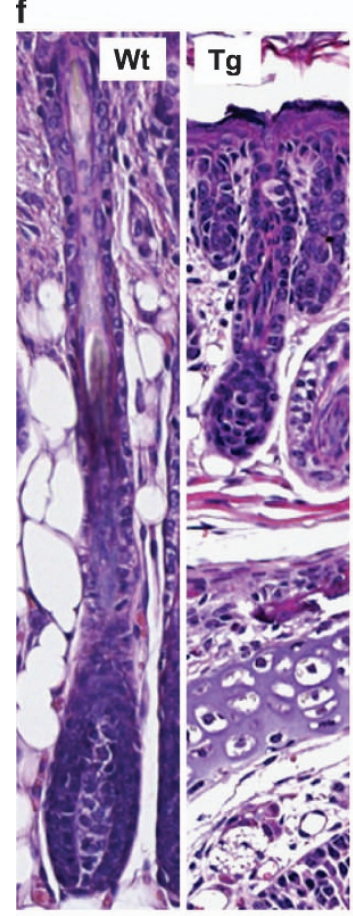

g
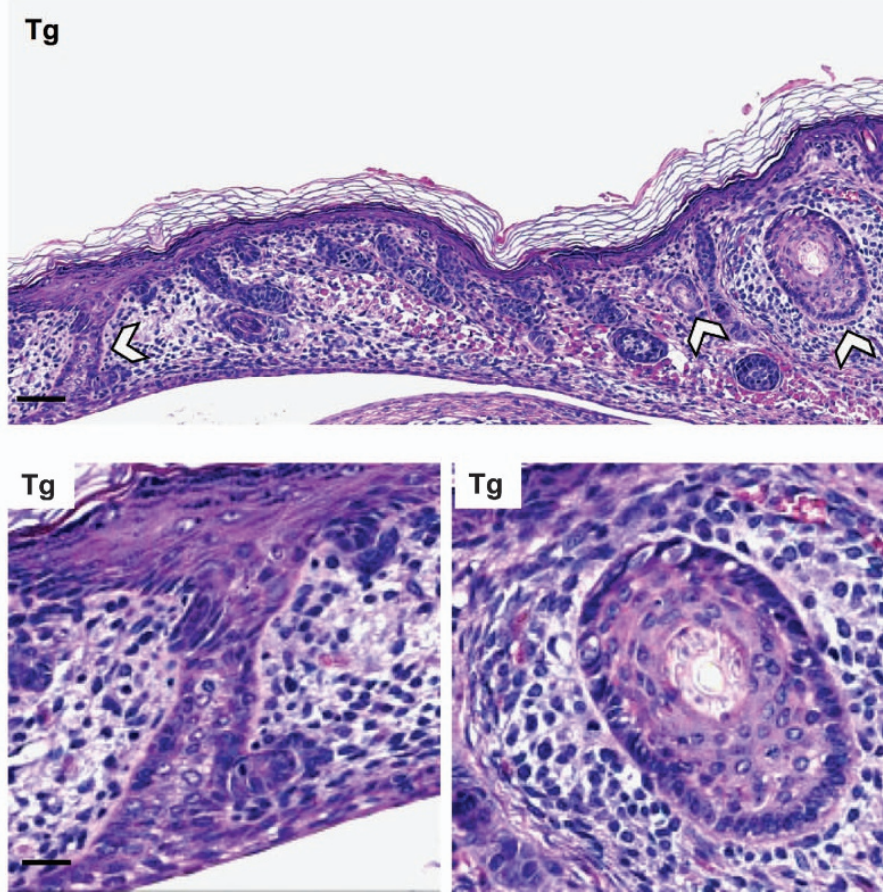

Figure 2 miR-24 transgenic mice displayed severe defects in hair follicle morphogenesis. (a) Schematic representation of transgene vector cassette for Tg;K5::miR-24. (b and c) Gross morphology of K5::miR-24 transgenic mice at 10 days post birth. The arrows in $\mathbf{c}$ indicate spots where hair coat is absent. (e) Histogram quantification of hair follicle density per square centimeter in transgenic and wt. ( $\mathbf{d}, \mathbf{f}$ and $\mathbf{g}$ ) Hematoxylin-eosin staining of back skin showing abnormal hair follicle morphology in P3 transgenic mice. Black arrows in D indicate a hair follicle that fails to penetrate in dermal tissue. White arrows indicate cyst-like hair follicles. Bars indicate the following: $\mathbf{d}, 200 \mu \mathrm{m}$; f, $50 \mu \mathrm{m} ; \mathbf{g}, 200$ and $50 \mu \mathrm{m}$

the length of the HFs was significantly less than wt, the stratification of the inner sheets appeared reduced and the hair bulbs, where proliferating cells are maintained, were smaller (Figure 2f). The HF orientation was altered; they were misangled and wavy (Figures $2 \mathrm{~d}-\mathrm{g}$ ). In the same area, follicles appeared to fail penetration in dermal tissue (Figure 2d, see black arrows). Tg;K5::miR-24 HFs at P3 were overall immature and underdeveloped. In addition, in some areas, cyst-like HFs were observed (Figure 2g, see arrows). Similar structures have been described in 
K14::DICER1 mutant mice, ${ }^{20,37}$ and suggest an alteration in the embryonic pathway of HF morphogenesis. The overall histological analysis, performed on HFs in transgenic mouse back skin proved of aberrant morphogenesis following miR-24 ectopic expression.

MiR-24 ectopic expression alters HF differentiation. To better understand the basis of Tg;K5::miR-24 HF abnormal morphogenesis, we performed a range of immunostaining to analyze the different compartments of the HFs. As shown in Figure 2, stratification of transgenic HFs appeared reduced; indeed, the expression of $\mathrm{K} 5$ and $\mathrm{K} 14$ that is usually confined in ORS was expanded to the inner layers. Most of the cells present in the HFs showed K5 and K14 positivity, suggesting an enlargement of the ORS and impairment in differentiation of HF cells (Figures 3a and b). Consistently, differentiation of hair cortex, medulla and cuticles, as assayed by hair keratin protein (AE13) and tricohyalins (AE15), revealed a marked reduction of the expression of differentiation markers in Tg;K5::miR-24 HFs as compared with wt (Figure 3c). Furthermore, in several HFs, AE13 immunostaining was detected in the matrix, whereas it was absent in the IRS, where it was physiologically expressed (Figure 3d). These results show that miR-24 overexpression in ORS produces an altered differentiation process in HF keratinocytes.

MiR-24 ectopic expression decreases the proliferation of HFs. In the epidermis and epidermal appendages, proliferation and differentiation are mutually exclusive programs, which are finely controlled by different factors. ${ }^{1,38,39}$ As the expression of $\mathrm{AE} 13$ in $\mathrm{HF}$ matrix of transgenic mice suggested an impaired control of proliferation versus differentiation, we analyzed the effects of miR-24 on proliferation capacity. Hence, we transfected primary human keratinocytes with pre-miR-24 and anti-miR-24, and we performed a 4-h 5-bromodeoxyuridine (BrdU)-pulse chase. Forty-eight and 72 hours of miR-24 overexpressions were able to repress $40 \%$ of the BrdU incorporation (Figure $4 a$ ), confirming that miR-24 strongly affects the proliferation potential. Anti-miR-24 did not affect proliferation; this was probably due to the non-relevant expression level of miR-24 in proliferating keratinocytes. Next, we analyzed the
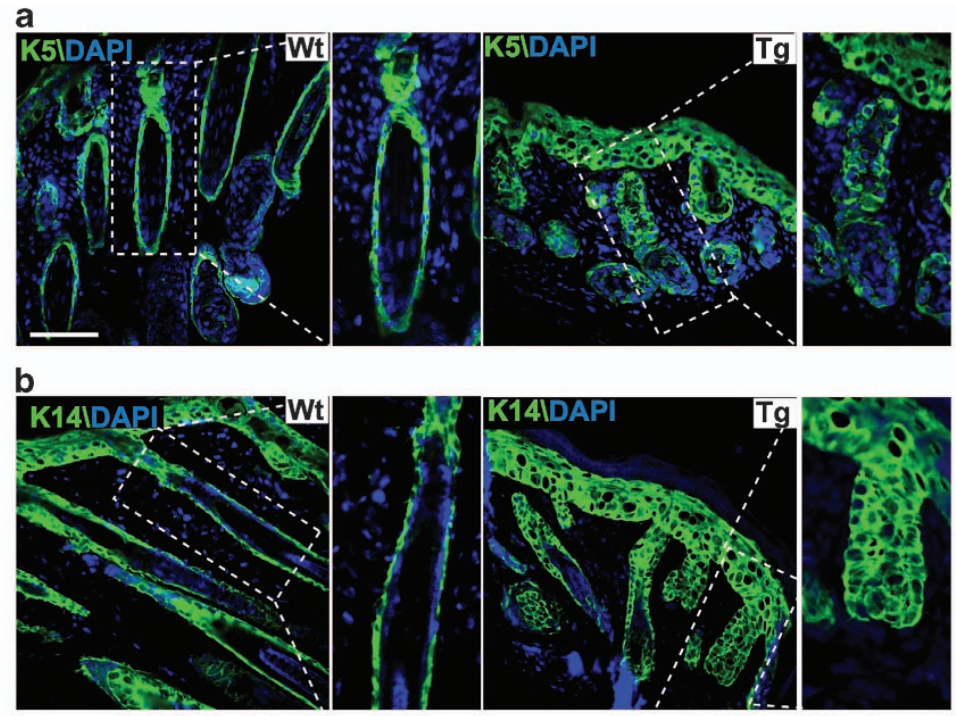

c

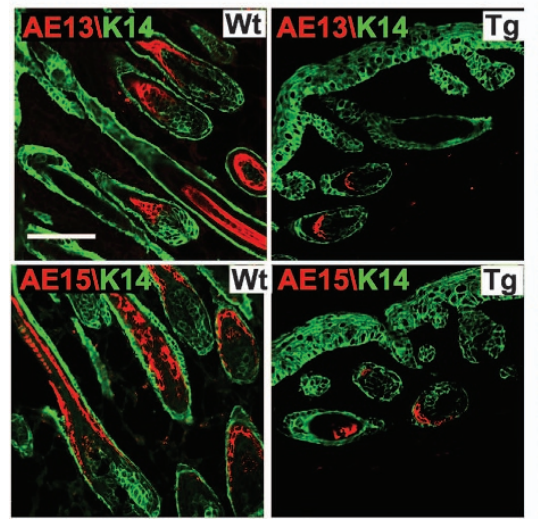

C
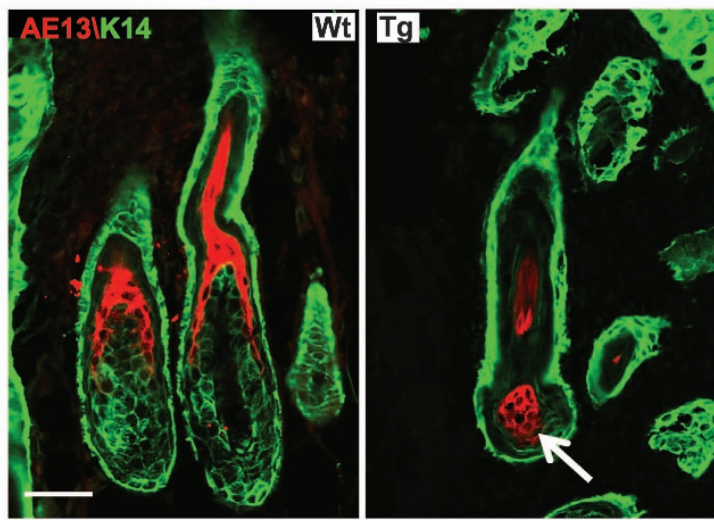

Figure 3 miR-24 transgenic mice displayed altered expression of differentiation markers in the hair follicle. (a and $\mathbf{b})$ Staining for cytokeratins $\mathrm{K} 5$ and $\mathrm{K} 14$, as markers of ORS, showing an extended expression in the inner layers of the transgenic hair follicle. Bars indicate $50 \mu \mathrm{m}$. (c and d) Hair keratin protein (AE13) and tricohyalin (AE15) staining (red) reveals a marked reduction of the expression of differentiation markers in transgenic hair follicles and ectopic expression in unphysiological compartment (see arrows). Bars indicate $50 \mu \mathrm{m}$ 


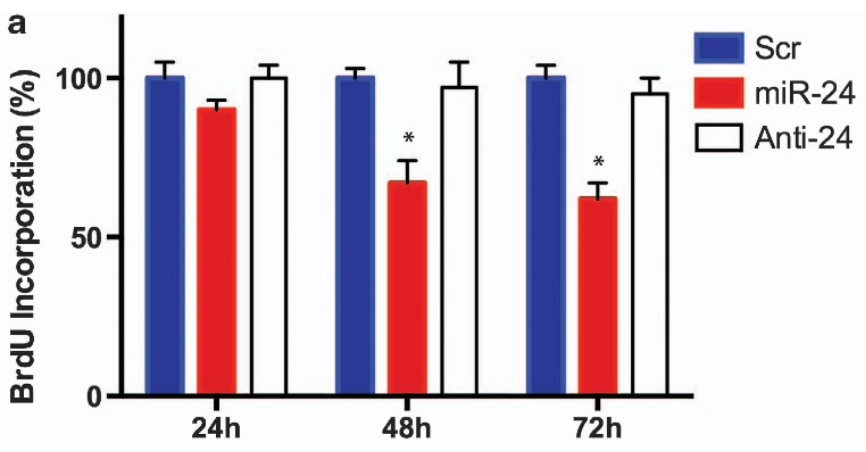

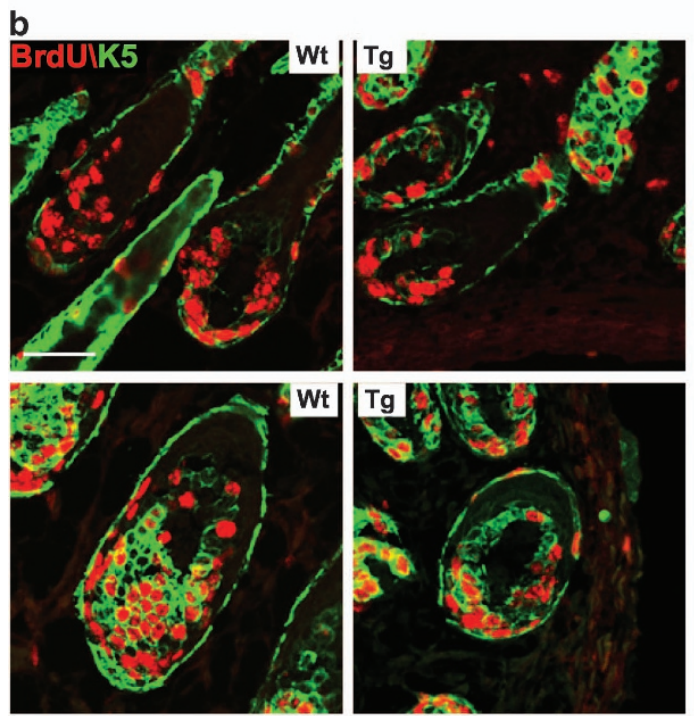
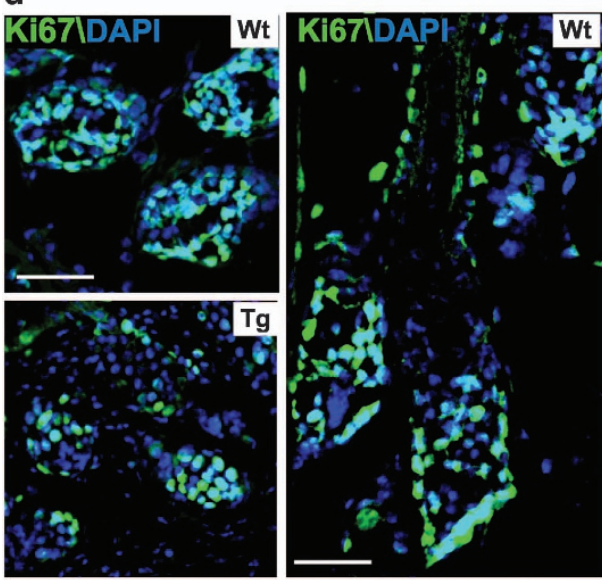
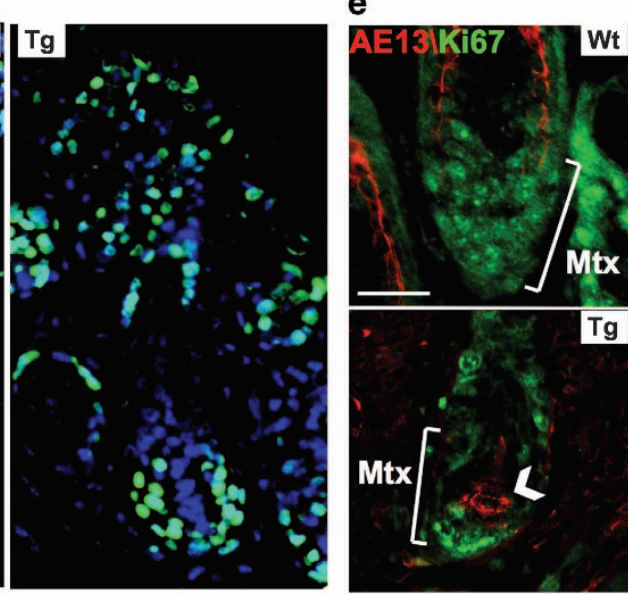

Figure 4 miR-24 transgenic mice show a significant reduction of the proliferation potential in hair follicles. (a) miR-24 overexpression reduces BrdU incorporation in ex vivo primary keratinocytes $\left({ }^{*} P<0.05\right)$. (c and $\left.\mathbf{b}\right)$ Ectopic expression of miR-24 reduced BrdU incorporation in P3 mouse hair follicle. Bar indicates $50 \mu \mathrm{m}$. Histograms show quantification of BrdU incorporation ( $\left.{ }^{\star} P<0.05\right)$. (d) Skin from transgenic mice showed reduced Ki67-positive cells. Bar indicates $50 \mu \mathrm{m}$. (e) Co-immunostaining for Ki67 (green) and AE13 (red) showed altered expression of differentiation markers of hair follicle. Mtx: Matrix. Bar indicates $40 \mu \mathrm{m}$

proliferation capacity of $\mathrm{HF}$ matrixes from $\mathrm{Tg}$ mice. We quantified the proliferating nuclei by BrdU incorporation experiments. The number of proliferating nuclei was significantly reduced in transgenic HFs (37\% reduction of the proliferating nuclei in transgenic mice) (Figures $4 \mathrm{~b}$ and $\mathrm{c}$ ). Defective proliferation was also confirmed by Ki67 staining. Ki67-positive cells appeared reduced in both vertical and longitudinal sections of the HF matrix (Figure 4d). To further correlate the reduction of proliferation to the early expression of differentiation markers, we performed a co-immunostaining of Ki67 and AE13. As shown in Figure 4e, the expression of $\mathrm{AE} 13$ in HF matrix of Tg;K5::miR-24 was concomitant to a reduction of Ki67 staining; no colocalization of $\mathrm{Ki} 67$ and AE13 was observed. These results confirm that K5-driven 
miR-24 expression in transgenic mice led to an early switch from proliferation to differentiation. Taken together, all these findings demonstrate that miR-24 ectopic expression alters normal cell fate of HF keratinocytes, leading to an aberrant HF morphogenesis, and suggesting an important role for miR-24 in HF development.

MiR-24 directly represses Tcf-3, a regulator of keratinocyte undifferentiation status. To investigate the mechanism of miR-24 contribution in HF morphogenesis, we screened miR-24 possible targets. Among the putative targets predicted by TargetScan $5.2,{ }^{40}$ we identified the miR-24-binding sites in the 3'-UTR of Tcf-3. Tcf-3 is a transcriptional factor that maintains skin progenitor cells in the undifferentiated state and has a central role in the regulation of morphogenesis of $\mathrm{HF}$ and adult hair cycle. ${ }^{16,41}$ TargetScan software predicted two binding sites for miR-24 in the Tcf-3 mRNA 3'UTR. However, both binding sites were among the poorly conserved ones in the evolutionary scale. According to previous reports, we performed a bioinformatic analysis by using the algorithm rna22, which does not require the seed match. ${ }^{42}$ Using this algorithm, we found that the two miR-24-binding sites were also conserved in mouse Tcf-3 $3^{\prime}$ UTR (Figure 5a).
To prove the direct binding of miR-24 on Tcf-3 $3^{\prime} U T R$ as a mechanism for its mRNA repress, we cloned the mouse 3'UTR of Tcf-3 downstream a luciferase reporter gene. Cotransfection of the reporter construct and miR-24 in SaOS-2 cells showed a reduction of $40 \%$ in luciferase activity compared with the control (Figure 5b). This demonstrated the ability of miR-24 to directly repress Tcf-3 expression. To further confirm miR-24 effect on Tcf-3, we performed a realtime qPCR on transgenic mouse whole epidermis and on miR24-overexpressing human keratinocytes. We found that in transgenic mice miR-24 reduced by more than $55 \%$ of the mRNA level of Tcf-3, whereas miR-24 overexpression in keratinocytes resulted in a reduction of about $40 \%$ (Figure $5 \mathrm{c}$ ). To complete the analysis, we also evaluated Tcf-3 protein level in transgenic mouse skin and in miR-24-overexpressing keratinocytes using western blot analysis (Figure 5d). Finally, we performed an in vivo miR-24 knockdown in newborn mice by subcutaneously injecting antago-miR-24. ${ }^{27} 24 \mathrm{~h}$ after the last injection, levels of miR-24 in the skin of treated mice showed a $60 \%$ reduction (Figure $5 \mathrm{e}$ ). Strikingly, miR-24 depletion in the skin strongly promoted the accumulation of Tcf-3 protein level (Figure 5f). Overall, our data proved that miR-24 represses Tcf-3 expression in both in vitro and in vivo
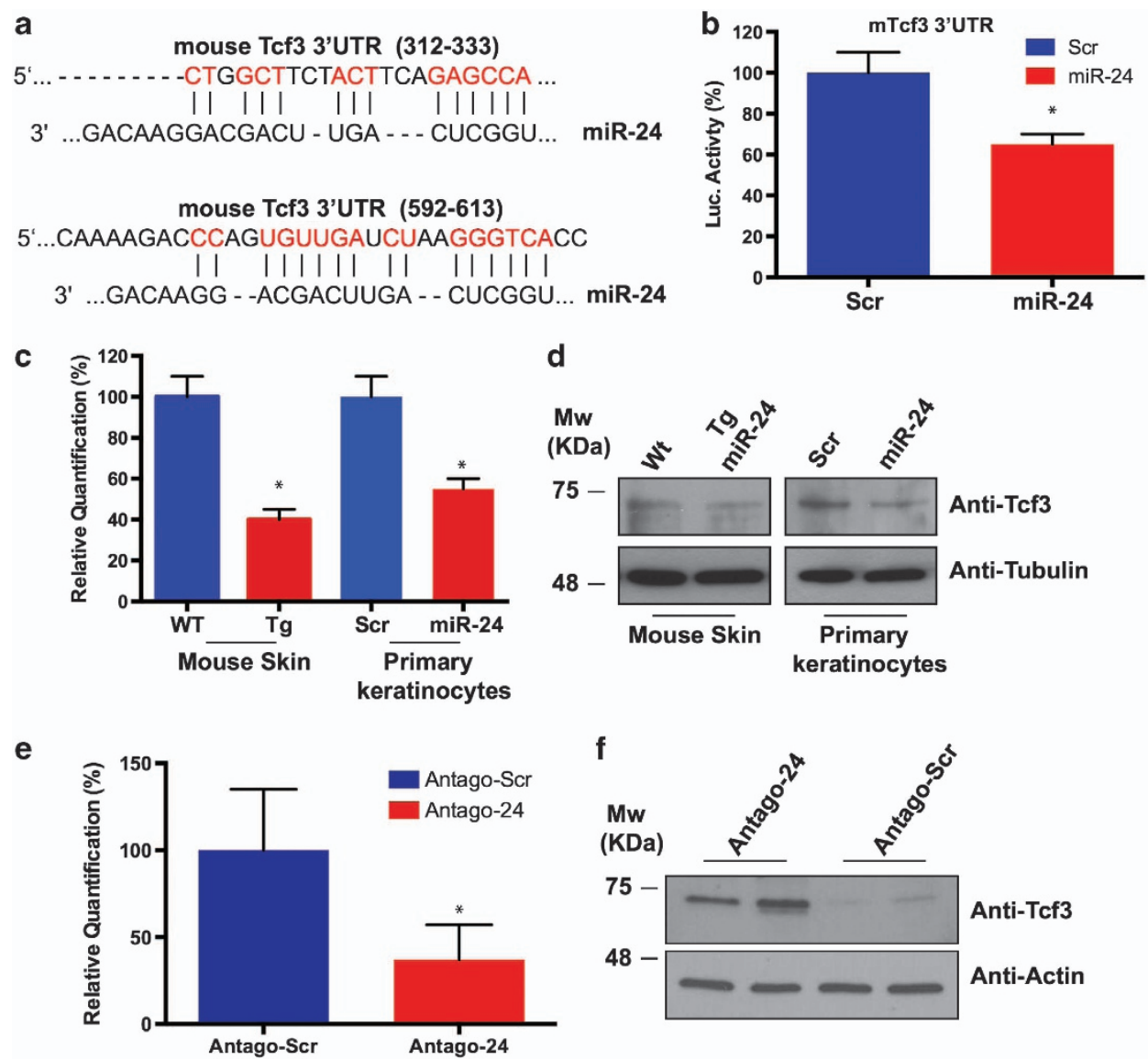

Figure 5 miR-24 directly represses Tcf-3 in vitro and in vivo. (a) 3'UTR from mouse and human Tcf-3 indicating the two putative miR-24 binding-sites predicted by the rna22 software. (b) Insertion of mouse Tcf-3 3'UTR decreases luciferase reporter activity. (c) qPCR shows in vitro and in vivo downregulation of Tcf-3 mRNA levels in miR-24overexpressing systems. (d) Western blot analysis in human primary keratinocytes $48 \mathrm{~h}$ post transfection or in P3 transgenic mice showed reduced Tcf-3 protein level. Tubulin was used as loading control. (e) Antago-miR-24 subcutaneous injection in newborn mice significantly reduced endogenous levels of miR-24 in the skin ( ${ }^{\star} P<0.01$ ). (f) Western blot analysis in Antago-24-treated mice showed increased levels of Tcf-3 in mouse skin. Actin was used as loading control 
models. Hence, miR-24/Tcf-3 axis provides a possible explanation for the HF phenotype of Tg;K5::miR-24.

MiR-24 ectopic expression depletes HF stem cells. If Tcf-3 is involved and it is a key target, we should expect a depletion of the stem cell compartment in the HFs of transgenic mice. To address this point, we performed confocal analysis on P3 mice skin to stain the stem cell compartment using $\mathrm{K} 15$ and Sox9. In contrast to the wt that presented a sustained and high level of $\mathrm{K} 15$ expression, there was a loss of $\mathrm{K} 15$ expression in transgenic mice HFs (Figure 6a). Similarly, immunostaining for Sox9 confirmed the marked depletion of Sox9-positive cells in transgenic mice HFs (Figure 6b). These findings supported our hypothesis of an miR-24 role in determining the switch from undifferentiated to differentiated state of HF stem cells. Therefore, overall, our data suggest a possible new role for miR-24 in HF supporting a link to the commitment of the HF stem cell.

\section{Discussion}

Morphogenesis and subsequent HF regeneration require a sustained stem cell compartment to ensure a constant refuel
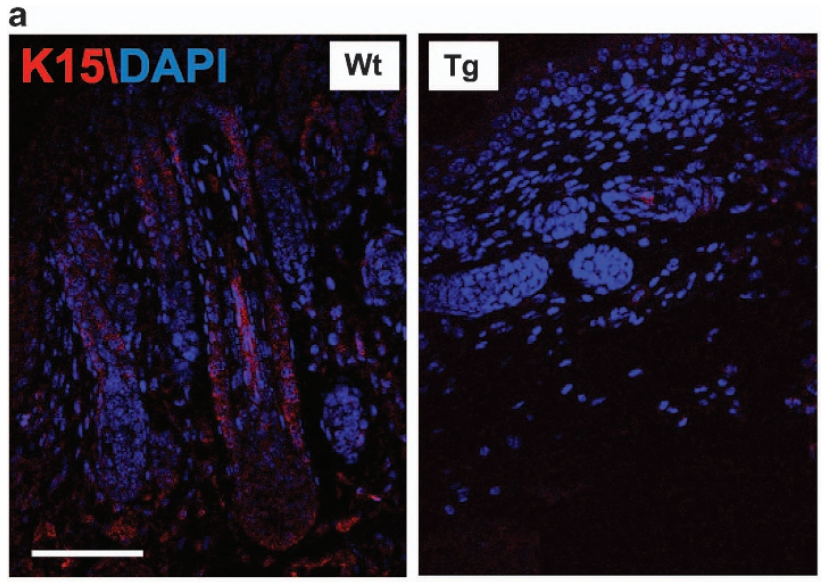

b
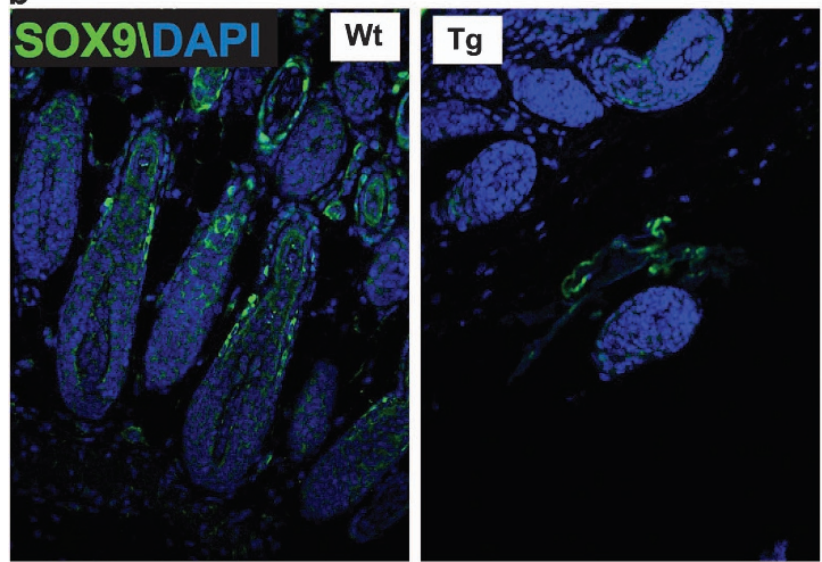

Figure 6 miR-24 affects stem cell marker expression of the hair follicle. (a and $\mathbf{b}$ ) Dorsal skin section from P3 mice stained with stem cell markers of hair follicle. As compared with wt control, the transgenic mice show a significant loss of K15 (red) or Sox9 (green) signal. Bars indicate $100 \mu \mathrm{m}$ of transient proliferating cells. HF stem cells express four key transcription factors: Sox9, Tcf-3, Lhx2 and NFATc1, which are responsible for maintenance of undifferentiated state. ${ }^{43}$ Mouse models, expressing mutants Sox9, Tcf-3, Lhx2 or NFATc1, showed depletion of stem cells in HFs. ${ }^{44-46}$ However, how these transcriptional factors are regulated during stem cell commitment is still only partially clear. Our data suggest a role for miR-24 in Tcf-3 regulation during the commitment of HF stem cells. miR-24 repressed the expression of Tcf-3 in both in vivo and in vitro models and showed the ability to directly bind Tcf-3 $3^{\prime} U T R$, resulting in its translational repression.

We conduced our study on Tg;K5::miR-24 transgenic mouse, which ectopically expresses miR-24 under the K5 promoter in HFs. miR-24 is mainly confined in the IRS; K5 promoter drove miR-24 expression in the ORS, where usually it is very weakly expressed. Thus, we generated a model with an early expression of the miRNA in the HF lineage differentiation. P3 transgenic mice showed a reduced number of HFs, with severe impairment of their morphogenesis. Analysis of specific markers revealed a failure of transgenic HFs to undergo proper differentiation. Indeed, we observed reduced proliferation, associated with premature expression of $A E 13$ differentiation marker in hair matrix. Lethality of the phenotype (mice die a few days after birth) limited further investigation of HF cycle in adult mice. However, the P3 mice phenotype showed a general accelerated differentiation and, consistent with previous reports, exposed a key role for miR24 in promoting HF differentiation. MiR-24, indeed, has been described to promote the differentiation of different cell types. In particular, it has been implicated in the differentiation of hematopoietic cell lines, ${ }^{28}$ myoblasts, ${ }^{31}$ neuronal cells ${ }^{29}$ and interfollicular keratinocytes. ${ }^{27}$ MiR-24 targets the $3^{\prime}$-UTR of two crucial cell cycle control genes, E2F2 and Myc, repressing their expression and resulting in a powerful anti-proliferative effect. $^{28}$ Hence, reduced proliferation in hair matrix of transgenic mice might be partially due to Myc downregulation. Myc is a main regulator of active hair cycling state by controlling the proliferation state of transient amplifying cells in HFs. ${ }^{45,47,48}$ However, altered terminal differentiation is not the only significant misregulation found in miR-24 transgenic HFs. We also identified a significant depletion of Sox9- and ${ }^{49} \mathrm{~K} 15$ positive cells, ${ }^{50}$ which might explain, at least in part, the abnormal HF morphogenesis. Strikingly, this phenotype may resemble the effect of Tcf-3/Tcf- 4 double deletion driven by skin-specific (Keratin 14) CRE recombinase. Tcf-3/Tcf-4 double conditional-null mice, indeed, display defects in epidermal homeostasis with the absence of hair coat, aberrant HFs and impaired stem cell maintenance. ${ }^{41}$ Tcf-3 has a pivotal role in maintenance of skin progenitor cells in the undifferentiated state. Tcf-3, indeed, is confined in HF 'bulge' region of adult epidermis or it is expressed in basal progenitors of the embryonic epidermis. ${ }^{10,16}$ Overexpression of Tcf-3 in the epidermis was shown to cause a reversion of adult cells to an embryonic-like status, associated with the accumulation of $\mathrm{K} 15$ and Sox9. Here, we demonstrate that miR-24 exerts a direct control on Tcf-3 expression. The analysis of the HF phenotype of miR-24 transgenic mice suggests a direct involvement of miR-24 in HF stem cell specification. The model we propose suggest that miR-24, 
repressing Tcf-3, can contribute to its shutdown that is required when premature undifferentiated stem cells are committed toward differentiation. MiR-24 progressively decreases Tcf-3 expression favoring HF stem cell activation and lineage specification, allowing constant refuel of transient proliferating cells in the matrix. Overall, we suggest a new function for miR-24 in contributing to the activation of epidermal stem cells, and at the same time we propose an additional mechanism for their commitment.

\section{Materials and Methods}

Transgenic mouse maintenance. $\mathrm{Tg} \mathrm{K5}:$ :miR-24 mice were generated in FVB mice strain as previously described. The PCR genotype was preformed with $5^{\prime}$ primer $5^{\prime}$-CCCCGCTGTAAAGAAAGATTTG- $3^{\prime}$ and $3^{\prime}$ primer $5^{\prime}$-CCCTTCATCT TCTTCTCCGTG-3'. Fifty milligram per kgof BrdU was administered in sterile PBS by intraperitoneal injection $4 \mathrm{~h}$ before killing the mice. Mouse colonies were maintained in the animal facility at the University of Rome Tor Vergata.

AntagomiR injection. Antago-mir-24 and scrambled control were designed and synthesized (Thermo Fisher Scientific, Waltham, MA, USA). The antago-mir-24 sequence was $5^{\prime}$-CpsUpsGUUCCUG-CUGAACUGAGpsCpsCpsAps-Chol-3' and scrambled control was $5^{\prime}$-UpsCps-ACAACCUCCUAGAAAGAGUpsApsGpsApsChol- $3^{\prime}$ (ps represents a phosphorothioate linkage, and Chol represents cholesterol linked through a hydroxyprolinol linkage). Subcutaneous injection was performed in newborn CD-1 mice at a dose of $80 \mathrm{mg} \mathrm{kg}^{-1}$ every day for 3 days.

Cell culture and transfection. Human primary epidermal keratinocytes from neonatal foreskin, HEKn (Cascade Biologics, Portland, OR, USA), were grown in EpiLife medium, supplemented with human keratinocyte growth supplement (HKGS) (Cascade Biologics, Invitrogen, Portland, OR, USA). Human primary keratinocytes were plated on collagen-coated dishes. Human primary keratinocytes were transfected with human pre-miR-24, anti-miR-24 and scramble negative control (Ambion, Austin, TX, USA) using the SiPORT neoFX transfection agent (Ambion) according to the manufacturer's protocols. The SAOS-2 cell line was grown in DMEM-F12 $(1: 1)$ medium, $10 \% \mathrm{FBS}, 100 \mathrm{U}$ penicillin and $100 \mu \mathrm{g}$ streptomycin (Gibco, Invitrogen, Carlsbad, CA, USA).

Proliferation assay. Human epidermal keratinocytes were pulse labeled for $4 \mathrm{~h}$ with $10 \mu \mathrm{M}$ of the BrdU analog EdU and then processed with the EdU Alexa Fluor 488 flow cytometry assay kit (Click-iT; Invitrogen, Carlsbad, CA, USA). Proliferating cells were analyzed and quantified with a flow cytometer (FACSCalibur; BD, Mountain View, CA, USA).

Real-time RT-PCR. Reverse transcription has been performed as previously described. Real-time PCR was performed using Platinum SYBR Green qPCR SuperMix UDG (Invitrogen) with the following amplification file: one cycle of $3 \mathrm{~min}$ at $95^{\circ} \mathrm{C}$ and 40 cycles of $20 \mathrm{~s}$ at $94^{\circ} \mathrm{C}$ and $40 \mathrm{~s}$ at $59^{\circ} \mathrm{C}$. The PCR reaction was monitored by a melting curve protocol according to the specification of the $A B I$ 7500 instrument (Applied Biosystems, Foster City, CA, USA). TBP mRNA was used as housekeeping gene for quantity normalization. Relative quantification of gene expression was calculated according to the method of $2^{-\Delta \Delta C t}$ described in the ABI User Bulletin no. 2 (updated in October 2001) and the RQ software version 1.3 of Applied Biosystems.

Western blotting. Total cell extracts were resolved in SDS-polyacrylamide gel and blotted onto Hybond PVDF membrane (GE Healthcare, Piscataway Township, NJ, USA). Membranes were blocked with PBST $5 \%$ non-fat dry milk, incubated with primary antibodies for $2 \mathrm{~h}$ at room temperature, washed and hybridized with peroxidase-conjugate secondary antibody for $1 \mathrm{~h}$ (rabbit, mouse and goat; Bio-Rad, Hercules, CA, USA). Detection was performed with the ECL chemiluminescence kit (Perkin Elmer, Waltham, MA, USA). The antibodies used were anti- $\beta$-tubulin, anti-actin and anti-Tcf-3 (Santa Cruz, Heidelberg, Germany and SIGMA, St. Louis, MO, USA).

Immunofluorescence and confocal analysis. Mouse tissues were embedded in frozen specimen medium Cryomatrix (Shandon, Waltham, MA, USA) or fixed in $4 \%$ paraformaldehyde $(48 \mathrm{~h})$ and embedded in paraffin. Nonspecific antigens were blocked by incubation in $5 \%$ goat serum in PBS for $1 \mathrm{~h}$ in a humidified atmosphere at room temperature. Subsequently, sections were incubated for $1 \mathrm{~h}$ with primary antibodies, anti-K14, anti-K10, (Covance; 1/1000 dilution), anti-BrdU (DAKO, Carpinteria, CA, USA, 1/50 dilution), anti-K15 (Thermo, 1\100), anti-Sox9 (Millipore, Billerica, MA, USA; $1 \backslash 100$ ), anti-AE13 and anti-AE15 (Santa Cruz, 1\100), and then washed three times with PBS and incubated for $1 \mathrm{~h}$ with 488- or 568-Alexa Fluor secondary antibodies, plus DAPI (Molecular Probe, Invitrogen, Carlsbad, CA, USA; dilution 1/1000). HematoxylinEosin staining was performed as previously described ${ }^{27}$ on paraffin-embedded sections to perform morphological analysis of tissue samples. Slides were then mounted by using the Prolong Antifade kit (Invitrogen), and fluorescence was evaluated by confocal microscopy (Nikon Instruments, Amsterdam, Netherlands; C1 on Eclipse Ti; EZC1 software) fitted with an argon laser (488-nm excitation), $\mathrm{He} / \mathrm{Ne}$ laser (542-nm excitation) and UV excitation at $405 \mathrm{~nm}$.

miRNA in situ hybridization analysis. Mouse tissues were embedded in frozen specimen medium Cryomatrix, after an overnight incubation in $4 \%$ paraformaldehyde, followed by an overnight incubation in $0.5 \mathrm{M}$ sucrose. Fourteen-micrometer-thick sections were cut and mounted on Superfrost glass slides. Slides were then fixed for $10 \mathrm{~min}$ in $4 \%$ paraformaldehyde and acetylate for $10 \mathrm{~min}$ in triethanolamine/acetic anhydride. Slides were then hybridized in incubation chambers overnight at $50^{\circ} \mathrm{C}$ using $30 \mathrm{nM}$ miRCURY LNA detection DIG-probes (Exiqon, Woburn, MA, USA). After hybridization, slides were washed (20 min in 5X SSC, two times for $30 \mathrm{~min}$ in $50 \%$ formamide/0.1 Tween-20/1 $\times$ SCC at $50^{\circ} \mathrm{C}$ and two times for $15 \mathrm{~min}$ in $0.2 \times \mathrm{SSC}, 15 \mathrm{~min}$ in PBS at RT). After $1 \mathrm{~h}$ of incubation in blocking solution (10\% goat serum $/ 0,1 \%$ Tween-20 in PBS) at RT, slides were hybridized for $2 \mathrm{~h}$ in AP-conjugated anti-DIG Fab fragment (Roche, Basel, Switzerland; $1 / 1500$ dilution) at RT. After two washes of 20 min, detection was performed by incubating $250 \mu$ of 1-STEP NBT/BCIP (Pierce, Rockford, IL, USA) together with $2 \mathrm{mM}$ Levamisole on a slide for $16 \mathrm{~h}$ in the dark at RT. Images of the areas were collected with an NIKON Eclipse TE200 microscope equipped with a NIKON DS Fi1 camera.

Luciferase assay and construct. A total of $2 \times 10^{5}$ SAOS-2 cells were seeded in 12-well dishes $24 \mathrm{~h}$ before transfection. One hundred nanograms of pGL3 vectors, $50 \mathrm{nM}$ of pre-miR-24 or scramble control and $10 \mathrm{ng}$ of pRL-CMV vector were cotransfected using Lipofectamine 2000 (Invitrogen). Luciferase activities of cellular extracts were measured $24 \mathrm{~h}$ after transfection, by using a Dual Luciferase Reporter Assay System (Promega, Madison, WI, USA); light emission was measured over $10 \mathrm{~s}$ using an OPTOCOMP I luminometer. Efficiency of transfection was normalized using Renilla luciferase activity. A fragment from mouse Tcf-3 $3^{\prime}$ UTR was amplified with $5^{\prime}$ primer $5^{\prime}$-GCTAGCAGAAAAGTTCCTT CCTTCCTCTCGG-3' and $3^{\prime}$ primer $5^{\prime}$-GCTAGCGGACTGCTGGCACACTGAATAG-3'.

Bioinformatics. miR-24 target sites on PAK4, Tks5 and ArhGAP19 3'UTR were predicted by the TargetScan 5.1 software available at http//www.targetscan. org and with rna22 available at http://cbcsrv.watson.ibm.com/rna22.html.

\section{Conflict of Interest}

The authors declare no conflict of interest.

1. Candi E, Schmidt R, Melino G. The cornified envelope: a model of cell death in the skin. Nat Rev Mol Cell Biol 2005; 6: 328-340.

2. Blanpain C, Fuchs E. Epidermal stem cells of the skin. Ann Rev Cell Dev Biol 2006; 22: 339-373.

3. Kaufman CK, Zhou P, Pasolli HA, Rendl M, Bolotin D, Lim KC et al. GATA-3: an unexpected regulator of cell lineage determination in skin. Genes Dev 2003; 17 : 2108-2122.

4. O'Guin WM, Sun TT, Manabe M. Interaction of trichohyalin with intermediate filaments: three immunologically defined stages of trichohyalin maturation. J Invest Dermatol 1992; 98: 24-32.

5. Tarcsa E, Marekov LN, Andreoli J, Idler WW, Candi E, Chung SI et al. The fate of trichohyalin. Sequential post-translational modifications by peptidyl-arginine deiminase and transglutaminases. J Biol Chem 1997; 272: 27893-27901.

6. Schneider MR, Schmidt-Ullrich R, Paus R. The hair follicle as a dynamic miniorgan. Curr Biol 2009; 19: R132-R142.

7. Truzzi F, Marconi A, Atzei P, Panza MC, Lotti R, Dallaglio K et al. p75 neurotrophin receptor mediates apoptosis in transit-amplifying cells and its overexpression restores cell death in psoriatic keratinocytes. Cell Death Differ 2011; 18: 948-958. 
8. Huelsken J, Vogel R, Erdmann B, Cotsarelis G, Birchmeier W. beta-Catenin controls hair follicle morphogenesis and stem cell differentiation in the skin. Cell 2001; 105: 533-545.

9. Andl T, Ahn K, Kairo A, Chu EY, Wine-Lee L, Reddy ST et al. Epithelial Bmpr1a regulates differentiation and proliferation in postnatal hair follicles and is essential for tooth development. Development 2004; 131: 2257-2268

10. Merrill BJ, Gat U, DasGupta R, Fuchs E. Tcf3 and Lef1 regulate lineage differentiation of multipotent stem cells in skin. Genes Dev 2001; 15: 1688-1705.

11. van Genderen C, Okamura RM, Farinas I, Quo RG, Parslow TG, Bruhn L et al. Development of several organs that require inductive epithelial-mesenchymal interactions is impaired in LEF-1-deficient mice. Genes Dev 1994; 8: 2691-2703.

12. Niemann C, Owens DM, Hulsken J, Birchmeier W, Watt FM. Expression of DeltaNLef1 in mouse epidermis results in differentiation of hair follicles into squamous epidermal cysts and formation of skin tumours. Development 2002; 129: 95-109.

13. Zhou $P$, Byrne $C$, Jacobs J, Fuchs $E$. Lymphoid enhancer factor 1 directs hair follicle patterning and epithelial cell fate. Genes Dev 1995; 9: 700-713.

14. Ahmad I, Patel R, Liu Y, Singh LB, Taketo MM, Wu XR et al. Ras mutation cooperates with beta-catenin activation to drive bladder tumourigenesis. Cell Death Dis 2011; 2: e124.

15. Petersson M, Brylka H, Kraus A, John S, Rappl G, Schettina P et al. TCF/Lef1 activity controls establishment of diverse stem and progenitor cell compartments in mouse epidermis. EMBO J 2011; 30: 3004-3018.

16. Nguyen $H$, Rendl M, Fuchs $E$. Tcf3 governs stem cell features and represses cell fate determination in skin. Cell 2006; 127: 171-183.

17. DasGupta R, Fuchs E. Multiple roles for activated LEF/TCF transcription complexes during hair follicle development and differentiation. Development 1999; 126: 4557-4568.

18. Merrill BJ, Pasolli HA, Polak L, Rendl M, Garcia-Garcia MJ, Anderson KV et al. Tcf3: a transcriptional regulator of axis induction in the early embryo. Development 2004; 131: 263-274

19. Aberdam D, Candi E, Knight RA, Melino G. miRNAs, 'stemness' and skin. Trends Biochem Sci 2008; 33: 583-591.

20. Andl T, Murchison EP, Liu F, Zhang Y, Yunta-Gonzalez M, Tobias JW et al. The miRNAprocessing enzyme dicer is essential for the morphogenesis and maintenance of hair follicles. Curr Biol 2006; 16: 1041-1049.

21. Lena AM, Shalom-Feuerstein R, Rivetti di Val Cervo P, Aberdam D, Knight RA, Melino G et al. miR-203 represses 'stemness' by repressing DeltaNp63. Cell Death Diff 2008; 15: 1187-1195.

22. Lena AM, Mancini M, Rivetti di Val Cervo P, Saintigny G, Mahe C, Melino G et al. MicroRNA-191 triggers keratinocytes senescence by SATB1 and CDK6 downregulation. Biochem Biophys Res Comm 2012; 423: 509-514.

23. Rivetti di Val Cervo P, Lena AM, Nicoloso M, Rossi S, Mancini M, Zhou $\mathrm{H}$ et al. p63-microRNA feedback in keratinocyte senescence. Proc Natl Acad Sci USA 2012; 109 1133-1138.

24. Viticchie G, Lena AM, Cianfarani F, Odorisio T, Annicchiarico-Petruzzelli M, Melino G et al. MicroRNA-203 contributes to skin re-epithelialization. Cell Death Dis 2012; 3: e435.

25. Teta M, Choi YS, Okegbe T, Wong G, Tam OH, Chong MM et al. Inducible deletion of epidermal Dicer and Drosha reveals multiple functions for miRNAs in postnatal skin. Development 2012; 139: 1405-1416.

26. Li X, Zhang J, Gao L, McClellan S, Finan MA, Butler TW et al. MiR-181 mediates cell differentiation by interrupting the Lin28 and let-7 feedback circuit. Cell Death Differ 2012 19: 378-386.

27. Amelio I, Lena AM, Viticchie G, Shalom-Feuerstein R, Terrinoni A, Dinsdale D et al. miR-24 triggers epidermal differentiation by controlling actin adhesion and cell migration. J Cell Biol 2012; 199: 347-363.

28. Lal A, Navarro F, Maher CA, Maliszewski LE, Yan N, O'Day E et al. miR-24 Inhibits cell proliferation by targeting E2F2, MYC, and other cell-cycle genes via binding to 'seedless' 3'UTR microRNA recognition elements. Mol Cell 2009; 35: 610-625.

29. Fukuda Y, Kawasaki H, Taira K. Exploration of human miRNA target genes in neurona differentiation. Nucleic Acids Symp Ser (Oxf) 2005; 49: 341-342.

30. Zimmer B, Kuegler PB, Baudis B, Genewsky A, Tanavde V, Koh W et al. Coordinated waves of gene expression during neuronal differentiation of embryonic stem cells as basis for novel approaches to developmental neurotoxicity testing. Cell Death Differ 2011; 18 : 383-395
31. Sun Q, Zhang Y, Yang G, Chen X, Zhang Y, Cao G et al. Transforming growth factorbeta-regulated miR-24 promotes skeletal muscle differentiation. Nucleic Acids Res 2008; 36: 2690-2699.

32. Paul C, Sardet C, Fabbrizio E. The histone- and PRMT5-associated protein COPR5 is required for myogenic differentiation. Cell Death Differ 2012; 19: 900-908.

33. Muller H, Bracken AP, Vernell R, Moroni MC, Christians F, Grassilli E et al. E2Fs regulate the expression of genes involved in differentiation, development, proliferation, and apoptosis. Genes Dev 2001; 15: 267-285.

34. Ingram L, Munro S, Coutts AS, La Thangue NB. E2F-1 regulation by an unusual DNA damage-responsive DP partner subunit. Cell Death Differ 2011; 18: 122-132.

35. Secombe J, Pierce SB, Eisenman RN. Myc: a weapon of mass destruction. Cell 2004; 117: 153-156.

36. Gramling MW, Eischen CM. Suppression of Ras/Mapk pathway signaling inhibits Myc-induced lymphomagenesis. Cell Death Differ 2012; 19: 1220-1227.

37. Yi R, O'Carroll D, Pasolli HA, Zhang Z, Dietrich FS, Tarakhovsky A et al. Morphogenesis in skin is governed by discrete sets of differentially expressed microRNAs. Nat Genet 2006; 38: $356-362$

38. Lena AM, Cipollone R, Amelio I, Catani MV, Ramadan S, Browne G et al. Skn-1a/Oct-11 and DeltaNp63alpha exert antagonizing effects on human keratin expression. Biochem Biophys Res Commun 2010; 401: 568-573.

39. Rufini S, Lena AM, Cadot B, Mele S, Amelio I, Terrinoni A et al. The sterile alpha-motif (SAM) domain of $\mathrm{p} 63$ binds in vitro monoasialoganglioside (GM1) micelles. Biochem Pharmacol 2011; 82: 1262-1268.

40. Lewis BP, Shih IH, Jones-Rhoades MW, Bartel DP, Burge CB. Prediction of mammalian microRNA targets. Cell 2003; 115: 787-798.

41. Nguyen H, Merrill BJ, Polak L, Nikolova M, Rendl M, Shaver TM et al. Tcf3 and Tcf4 are essential for long-term homeostasis of skin epithelia. Nat Genet 2009; 41: 1068-1075.

42. Miranda KC, Huynh T, Tay Y, Ang YS, Tam WL, Thomson AM et al. A pattern-based method for the identification of MicroRNA binding sites and their corresponding heteroduplexes. Cell 2006; 126: 1203-1217.

43. Nowak JA, Polak L, Pasolli HA, Fuchs E. Hair follicle stem cells are specified and function in early skin morphogenesis. Cell Stem Cell 2008; 3: 33-43.

44. Horsley V, O'Carroll D, Tooze R, Ohinata Y, Saitou M, Obukhanych T et al. Blimp1 defines a progenitor population that governs cellular input to the sebaceous gland. Cell 2006; 126 : 597-609.

45. Rhee H, Polak L, Fuchs E. Lhx2 maintains stem cell character in hair follicles. Science 2006; 312: 1946-1949.

46. Ouji Y, Ishizaka S, Nakamura-Uchiyama F, Yoshikawa M. In vitro differentiation of mouse embryonic stem cells into inner ear hair cell-like cells using stromal cell conditioned medium. Cell Death Dis 2012; 3: e314.

47. Hara T, Miyazaki M, Hakuno F, Takahashi S, Chida K. PKCeta promotes a proliferation to differentiation switch in keratinocytes via upregulation of p27Kip1 mRNA through suppression of JNK/c-Jun signaling under stress conditions. Cell Death Dis 2011; 2: e157.

48. Liu B, Willette-Brown J, Liu S, Chen X, Fischer SM, Hu Y. IKKalpha represses a network of inflammation and proliferation pathways and elevates $\mathrm{c}-M y c$ antagonists and differentiation in a dose-dependent manner in the skin. Cell Death Differ 2011; 18: 1854-1864.

49. Vidal VP, Chaboissier MC, Lutzkendorf S, Cotsarelis G, Mill P, Hui CC et al. Sox9 is essential for outer root sheath differentiation and the formation of the hair stem cell compartment. Curr Biol 2005; 15: 1340-1351.

50. Troy TC, Arabzadeh A, Turksen K. Re-assessing K15 as an epidermal stem cell marker. Stem Cell Rev 2011; 7: 927-934.

(c) (i) $(\theta)$ Cell Death and Disease is an open-access journal published by Nature Publishing Group. This work is licensed under a Creative Commons Attribution-NonCommercialNoDerivs 3.0 Unported License. To view a copy of this license, visit http://creativecommons.org/licenses/by-nc-nd/3.0/ 